\title{
Isolation, Identification, and Antibiotic Susceptibility Testing of Salmonella from Slaughtered Bovines and Ovines in Addis Ababa Abattoir Enterprise, Ethiopia: A Cross-Sectional Study
}

\author{
Abe Kebede, ${ }^{1,2}$ Jelalu Kemal, ${ }^{1}$ Haile Alemayehu, ${ }^{2}$ and Solomon Habte Mariam ${ }^{2}$ \\ ${ }^{1}$ Haramaya University College of Veterinary Medicine, P.O. Box 138, Dire Dawa, Ethiopia \\ ${ }^{2}$ Aklilu Lemma Institute of Pathobiology, P.O. Box 1176, Addis Ababa, Ethiopia \\ Correspondence should be addressed to Solomon Habte Mariam; solomon.habtemariam@aau.edu.et
}

Received 9 June 2016; Revised 12 July 2016; Accepted 25 July 2016

Academic Editor: Gary Dykes

Copyright (C) 2016 Abe Kebede et al. This is an open access article distributed under the Creative Commons Attribution License, which permits unrestricted use, distribution, and reproduction in any medium, provided the original work is properly cited.

\begin{abstract}
Salmonellae are ubiquitous, found in animals, humans, and the environment, a condition which facilitates transmission and cross contamination. Salmonella enterica serotypes exert huge health and economic impacts due to their virulence or carriage of antibiotic resistance traits. To address this significant issues with regard to public health, availability of adequate information on the prevalence and antibiotic resistance patterns of Salmonella, and establishment of adequate measures to control contamination and infection are needed. A cross-sectional study was conducted to assess the level of Salmonella infection in slaughtered bovines and ovines at Addis Ababa abattoir. Samples were collected randomly and processed for identification and antimicrobial susceptibility testing of Salmonella spp. From 280 animals examined, 13 (4.64\%) (8 bovines and 5 ovines) were positive, with most samples (12/13, 92\%) comprising Salmonella Dublin. Very high level of resistance to some antibiotics used in human medicine was detected. Most isolates were susceptible to gentamycin and amikacin. Nine (69\%) of all isolates were resistant to multiple antibiotics. Serotyping revealed 12 of 13 isolates to be of the Dublin serotype with 9,12:g,p:- antigenic formula. This study emphasizes the importance of improving the evisceration practice during slaughtering and restricting the use of antibiotics in farm animals.
\end{abstract}

\section{Introduction}

Globally, Salmonella has been one of the most commonly reported causes of food-borne pathogens from distant and recent times [1-3]. According to a recent study [4] commissioned by the World Health Organization (WHO) on the global disease burden of food-borne diseases in humans, food-borne illnesses from diarrheal and invasive nontyphoidal Salmonella enterica, resulted in the largest disease burden, highlighting the significant public health importance of Salmonella infections and the urgency for control, particularly in low- and middle-income countries where most burden of diseases and occurrence of mortality cases are reported. In sub-Saharan Africa, nontyphoidal salmonellae are the most common causes of bacterial bloodstream infections in both adults and children presented with fever and are associated with case fatality rate of 20-25\% [5]. Infections can occur most often via ingestion of contaminated meat, eggs, raw milk, fruits, and vegetables [6-8]. Contamination of these foods can occur during production, processing, distribution, and retail marketing [9]. Nontyphoidal salmonellae, including $S$. Dublin, are known to cause bacteremia and other infections in humans in sub-Saharan Africa [10-13]. S. Dublin is primarily cattle-adapted, but it can also less frequently cause infections in other domestic animals, including ovines [14, 15]. Food-producing animals, including bovines and ovines, serve as reservoirs of nontyphoidal Salmonella serotypes that can be transmitted to humans [16, 17]. Wild animals can also serve as reservoirs of Salmonella increasing its transmittance to free-ranging food animals and then to humans through cross contamination $[18,19]$.

An increasing proportion of Salmonella isolates is resistant to commonly used antibiotics in both developing and developed countries [20,21], and this increase is seen in both veterinary and public health sectors [22-24]. The increasing proportion of single and multiple antibiotic-resistant 
Salmonella strains isolated from human salmonellosis cases has been associated with the widespread use of antibiotics in food animals [25]. A recent review [26] indicated that, overall, several factors contribute to high antibiotic resistance in Ethiopia, including ease of access to and high frequency of antibiotic use, use of antibiotics at subtherapeutic levels, overprescription at health facilities, close contact between animals, high antibiotic use in animals in small production systems, and contamination during handling animal products; but another study indicated the exact extent of use of antimicrobials in food animals in Ethiopia is not clearly defined [25]. However, levels of antibiotics in beef have been found to be high in Ethiopia [27]. The antibiotics excessively used in Ethiopia and other African countries include tetracyclines, $\beta$-lactams, chloramphenicol, quinolones, nitrofurans, and macrolides. Tetracycline levels have been found to be especially high in meat and kidney samples from several abattoirs in Ethiopia, exceeding the WHO limits [27].

Contamination can occur at various levels. For example, fecal excretion of Salmonella can be a source of contamination both at farm and at abattoir levels. Contaminated hides and viscera can be sources of contamination at abattoirs. Abattoir workers can spread the contamination during evisceration and handling meat without proper hand disinfection. Some studies carried out on meat samples, minced meat, meat swab, and humans in Ethiopia showed that Salmonella is quite prevalent in various food animals (e.g., bovines, ovines, poultry, and pigs), animal products (e.g., beef, poultry, and milk), and human beings [28-32]. Animals and humans get Salmonella contamination in several ways. Animals get infected with Salmonella via the fecal-oral route through consumption of feeds, water, grass, and so forth, contaminated with feces from other infected animals, as well as through direct contact with infected animals. Salmonella can colonize animals at various sites, such as the intestines of food animals and the reproductive tract and egg of chicken, leading to contamination of various animal products. Humans become infected with Salmonella after consuming raw or improperly cooked animal products, such as contaminated meat, poultry, pork, and milk, as well as through direct contact with contaminated animals and household pets $[16,17,33,34]$.

In Ethiopia, as in other developing countries, it is difficult to evaluate the burden of food-borne diseases, because of the limited scope of studies and lack of coordinated epidemiological surveillance systems. In addition, underreporting of cases and the presence of other diseases considered to be of higher priority may have overshadowed the problem of food-borne diseases including salmonellosis. Therefore, the objectives of this study were to isolate and identify Salmonella in slaughtered bovines and ovines, estimate the prevalence, and investigate the susceptibility pattern of isolates to commonly used antibiotic agents using disk diffusion method.

\section{Materials and Methods}

2.1. Study Site Description and Duration. The study was conducted from December 2014 to April 2015 at Addis Ababa Abattoir Enterprise, Ethiopia. Addis Ababa is the capital city of Ethiopia. It lies in the central highlands at an altitude of
$2324 \mathrm{~m}(7625 \mathrm{Ft})$ above sea level. The annual average maximum and minimum temperatures are $26^{\circ} \mathrm{C}$ and $11^{\circ} \mathrm{C}$, respectively. The population size of the city is estimated at 3 million. The city's main supply of meat is provided by slaughter of bovines drawn from all corners of Ethiopia.

2.2. Bovine and Ovine Sampling. Healthy bovines and ovines were slaughtered at Addis Ababa abattoir enterprise after an average of 24-72 h upon arrival at the slaughterhouse. Feed and water were provided to the animals until $12 \mathrm{hrs}$ before slaughter. Most of the bovines slaughtered at the abattoir are adult males of local zebu breed. Currently, on average, 1200 bovines and 1000 ovines are slaughtered every day. Different organs and carcasses are inspected after slaughter, mainly for presence of tuberculous lesions, whereas no tests are conducted to detect Salmonella and other pathogens.

2.3. Sample Size Determination. For isolation and identification of Salmonella, the sample size was calculated based on 8.5\% [35] and 7.7\% [36] expected prevalence in bovine and ovine samples, respectively, with 5\% desired absolute precision and 95\% confidence interval using the formula recommended by Thrusfield [37]:

$$
n=\frac{Z^{2} \times p_{\exp }\left(1-p_{\exp }\right)}{d^{2}}
$$

where $n$ is required sample size, $Z$ is $1.96, p_{\exp }$ is expected prevalence, and $d$ is desired absolute precision of 0.05 .

Accordingly, the minimum sample size calculated was 120 and 109. To increase the precision of the estimate, the sample size was inflated and a total of 280 samples were considered. These samples included 140 from bovines (70 from carcass swab and 70 [35 each] from lung and liver) and 140 from ovines (70 from carcass swab and 70 [35 each] from lung and liver).

2.4. Study Design and Sampling Analysis. A cross-sectional study was conducted on randomly selected samples collected from Addis Ababa abattoir. Samples were collected using random sampling properly labelled by date of collection, sources, and sample type. The lung and liver samples were collected immediately after slaughter. Twenty-five grams of liver and lung samples was put in sterile plastic bags and kept in an ice box containing ice packs. The sampling areas were delineated by using sterile aluminum foil templates $(10 \times 10 \mathrm{~cm})$. A sterile cotton tipped swab $(2 \times 3 \mathrm{~cm})$ fitted with wooden shaft was first soaked in $10 \mathrm{~mL}$ of sterile buffered peptone water (BPW) (OXOID, England) and swabbed over the delineated area horizontally and then vertically several times on the abdomen (flank), thorax (lateral), crutch, and breast (lateral). Up on completion of the swabbing process, the swab was placed into the BPW within test tube and the upper tip of the shaft was broken and disposed of leaving the cotton swab [38]. The samples were kept in ice box containing ice packs and immediately transported to the Microbiology Laboratory at Aklilu Lemma Institute of Pathobiology. The samples were processed upon arrival. 


\subsection{Isolation and Identification}

2.5.1. Culture Methods, Plating, and Identification. The technique recommended by the International Organization for Standardization ISO 6579 [39] was employed in order to isolate and identify Salmonella organisms. Overnight frozen samples were allowed to thaw for 3 to $5 \mathrm{hrs}$ at room temperature before analysis. The bacteriological media used for the study were prepared following the instructions of the manufacturer. Each $25 \mathrm{~g}$ of sample was put in a sterile Stomacher Bag and $225 \mathrm{~mL}$ of buffered peptone water (OXOID, England) was added (one to nine proportion) and homogenized using a laboratory blender (OXOID, England) for 2 minutes. The preenriched samples were incubated for 18 to $24 \mathrm{hrs}$ at $37^{\circ} \mathrm{C}$. Following this, $1 \mathrm{~mL}$ and $0.1 \mathrm{~mL}$ of the preenrichment broths were transferred aseptically into $10 \mathrm{~mL}$ of Muller Kauffmann Tetrathionate (Merck, Germany) and $10 \mathrm{~mL}$ of RappaportVassiliadis (RV) broth (OXOID, England), mixed, and then were incubated for 18 to $24 \mathrm{hrs}$ at $37^{\circ} \mathrm{C}$ and $42^{\circ} \mathrm{C}$, respectively. Following incubation, a loop-full of each culture was streaked onto the surface of xylose lysine deoxycholate (XLD) (OXOID, England) and brilliant green agar (BGA) (OXOID, England) medium and incubated at $37^{\circ} \mathrm{C}$ for 24 to $48 \mathrm{hrs}$.

The XLD and BGA plates were examined for the presence of Salmonella colonies. If growth is slight or if typical colonies of Salmonella were not present, the plates were reincubated for a further 18 to $24 \mathrm{hrs}$ and reexamined for the presence of typical Salmonella colonies. The formation of red colonies with black centers and of pink colonies with a red zone was inspected on XLD and BGA plates, respectively [40].

Identification of isolates was initiated by Gram staining. All Gram-negative isolates were further identified by motility test (by the hanging-drop method), biochemical tests, PCR amplification, and serotyping.

2.5.2. Biochemical Tests. All suspected Salmonella colonies were picked from the agar plates and inoculated into the following biochemical test tubes for confirmation: triple sugar iron (TSI) test (presumptive Salmonella colonies produce black colonies or colonies with black centers and red medium on TSI agar) (OXOID, England), citrate test (presumptive Salmonella colonies produce blue colour for the citrate test), urease test (presumptive Salmonella colonies produce purple-red colour for the urease test), lysine decarboxylase (LDC) agar (OXOID, England) test (presumptive Salmonella colonies produce purple-coloured colonies on LDC agar), and indole test (presumptive Salmonella colonies produce violet-coloured colonies for the indole test). Plates were incubated for 24 or $48 \mathrm{hrs}$ at $37^{\circ} \mathrm{C}$ [41]. Colonies were also tested for catalase production.

2.5.3. Molecular Identification and Serotyping. A genusspecific polymerase chain reaction (PCR) amplification targeting histidine transport operon using pairs of primers 5'ACTGGCGTTATCCCTTTCTCTGGTG3' and 5'ATGTTGTCCTGCCCCTGGTAAGAGA3 ${ }^{\prime}$ was conducted to test the identity of the bacterial isolates using PCR conditions as described in Cohen et al. [42]. The bacterial isolates positive by the genus-specific PCR were serotyped by slide agglutination test targeting specific flagellar antigens.

2.6. Antibiotic Susceptibility Tests. The disc diffusion test was done for each isolate on Mueller-Hinton agar (OXOID, England). Approximately $20 \mathrm{~mL}$ of medium was poured into $90 \mathrm{~mm}$ diameter sterile Petri dishes to a depth of $4 \mathrm{~mm}$ and left at $37^{\circ} \mathrm{C}$ overnight to check for sterility. Five $\mathrm{mL}$ tryptic soya broth (OXOID, England) was inoculated with test isolates and incubated at $35^{\circ} \mathrm{C}$ for $4 \mathrm{hr}$. Culture of each isolate was compared with $0.5 \mathrm{McF}$ arland turbidity standards (if necessary adjusted by adding sterile saline into tubes). Isolates were inoculated on Mueller-Hinton agar using swabs and inoculated plates were left at room temperature for $30 \mathrm{~min}$ to allow drying. Salmonella isolates were tested for susceptibility to the following 14 antibiotics (OXOID, England): amikacin (10 $\mu \mathrm{g})$, amoxicillin/clavulanic acid $(30 \mu \mathrm{g})$, ampicillin $(10 \mu \mathrm{g})$, ceftriaxone $(30 \mu \mathrm{g})$, chloramphenicol $(30 \mu \mathrm{g})$, ciprofloxacin $(30 \mu \mathrm{g})$, gentamycin $(10 \mu \mathrm{g})$, kanamycin $(30 \mu \mathrm{g})$, nalidixic acid $(30 \mu \mathrm{g})$, nitrofurantoin $(30 \mu \mathrm{g})$, streptomycin $(10 \mu \mathrm{g})$, trimethoprim/sulfamethoxazole, and tetracycline $(30 \mu \mathrm{g})$ using the disk diffusion method according to guidelines set by the Clinical Laboratory Standards Institute (CLSI) [43]. Antibiotic impregnated discs were dispensed on the surface of cultures of Muller-Hinton agar and incubated at $35^{\circ} \mathrm{C}$ for $20 \mathrm{hrs}$. The diameters of the zones of inhibition were recorded to the nearest $\mathrm{mm}$ and classified as resistant, intermediate, or susceptible according to established interpretive chart [43].

2.7. Quality Control. The following quality control strains were used: Escherichia coli ATCC 25922 for positive control and Proteus mirabilis ATCC 35659 for negative control in the LDC and indole tests; Klebsiella pneumoniae ATCC 700603 for positive control and E. coli ATCC 25922 and uninoculated for negative control in the citrate and urease tests; and Salmonella typhimurium ATCC 14028 were used. K. pneumoniae ATCC 700603 and E. coli were used as positive and negative controls, respectively, for the citrate tests. The sterility of prepared media was checked by incubating some randomly selected plates for $24 \mathrm{hrs}$ at $37^{\circ} \mathrm{C}$. E. coli ATCC 25922 and K. pneumoniae ATCC 700603 were used as positive and negative controls in the motility test. E. coli ATCC 25922 was used as a reference strain in the disk diffusion susceptibility tests.

2.8. Data Analysis. Data collected in the study and the results of laboratory investigations were entered into Microsoft Excel, edited, coded, and analyzed by statistical methods using statistical software program (SPSS version 15.0). Percentage to measure the prevalence of Salmonella was used and $P$ value $<0.05$ was considered significant and Chi-square test was used to compute the association between explanatory variables.

\section{Results}

3.1. Prevalence of Salmonella in Samples. Among a total of 280 samples examined for bacteriological status, 19 bovine and ovine samples were positive for Salmonella by biochemical testing (but the number of true positive samples was reduced 
TABLE 1: Prevalence of Salmonella based on species and sample type from Addis Ababa abattoir.

\begin{tabular}{|c|c|c|c|c|c|c|c|c|c|c|}
\hline \multirow{2}{*}{ Sample } & \multicolumn{3}{|c|}{ Bovine } & \multicolumn{3}{|c|}{ Ovine } & \multirow{2}{*}{ Total } & \multirow{2}{*}{ Positive (\%) } & \multirow{2}{*}{$\chi^{2}$} & \multirow{2}{*}{$P$ value } \\
\hline & Number examined & Number positive & Percent & Number examined & Number positive & Percent & & & & \\
\hline Carcass swab & 70 & 4 & 5.7 & 70 & 1 & 1.4 & 140 & $5(3.5)$ & & \\
\hline Lung & 35 & 2 & 5.7 & 35 & 1 & 2.8 & 70 & $3(4.3)$ & 1.37 & 0.50 \\
\hline Liver & 35 & 2 & 5.7 & 35 & 3 & 8.5 & 70 & $5(7.1)$ & & \\
\hline Total & 140 & 8 & 5.7 & 140 & 5 & 3.57 & 280 & $13(4.64)$ & & \\
\hline
\end{tabular}

TABle 2: Serotype and antigen types of Salmonella isolates from bovine and ovine samples.

\begin{tabular}{ccccc}
\hline \multicolumn{6}{c}{ Species Sample ID } & Sample source & Serotype & Antigen \\
\hline & B-17 & Liver & Dublin & 9,12:g,p:- \\
& B-21 & Liver & Dublin & 9,12:g,p: \\
& B-48 & Lung & Dublin & 9,12:g,p: \\
Bovine & B-61 & Lung & Dublin & 9,12:g,p: \\
& BS-1 & Carcass swab & Dublin & 9,12:g,p: \\
& BS-12 & Carcass swab & Dublin & 9,12:g,p: \\
& BS-13 & Carcass swab & Dublin & 9,12:g,p: \\
& BS-23 & Carcass swab & Dublin & 9,12:g,p: \\
\hline \multirow{6}{*}{ Ovine } & L-6 & Liver & Dublin & 9,12:g,p: \\
& M-26 & Liver & Dublin & 9,12:g,p: \\
& M-28 & Liver & Dublin & 9,12:g,p: \\
& M-72 & Lung & I:ROUGH-O:g,p:- & -:g,p:- \\
& SS-16 & Carcass swab & Dublin & 9,12:g,p: \\
\hline
\end{tabular}

to 13 by further tests as described in Section 3.2). The isolates from bovine and ovine samples were all Gram-negative rods and motile. The samples were positive for the citrate, LDC, and $\mathrm{H}_{2} \mathrm{~S}$ production tests. The urease and indole tests were negative for these isolates. The isolates were positive for the catalase test.

3.2. Molecular and Serotype Identification of Salmonella. The PCR amplifications gave products of $496 \mathrm{bp}$ for 13 isolates. This is the expected size for samples positive for Salmonella by the genus-specific PCR reaction applied here [42]. Serotyping also revealed the same 13 isolates to be Salmonella spp. Out of these positive samples, $8(61.5 \%)$ were obtained from bovine samples and $5(38.5 \%)$ were from ovine samples. Salmonella was isolated from 2 lung, 2 liver, and 4 carcass swab samples of bovines and from 1 lung, 3 liver, and 1 carcass swab samples of ovines (Table 1). Twelve of 13 Salmonella-positive samples were found to be serotype Dublin and had the 9,12:g,p:antigenic epitope for both bovine and ovine samples. The other Salmonella isolates of ovine origin had the I:RoughO:g,p:- serotype (Table 2).

3.3. Antibiotic Susceptibility Tests. Single and multiple resistance to most of the antibiotics tested were observed. The highest level of resistance observed was streptomycin (100\%) with all 13 isolates resistant to streptomycin. The next highest resistance was to amoxicillin, with 10/13 isolates (77\%) being resistant. Gentamycin and amikacin were the most effective antibiotics, except that 1 (B.21) and 2 (B.S.23 and S.S.16) isolates were intermediate-resistant to the two antibiotics, respectively (Table 3 ). Ninety-two percent (12/13) of the isolates were found to be susceptible to gentamycin, while $85 \%$ (11/13) were susceptible to amikacin (Table 3 and see Supplementary Table S1 in Supplementary Material available online at http://dx.doi.org/10.1155/2016/3714785). The disk contents for all antibiotics we used were the same as those described under "Zone Diameter and Minimal Inhibitory Concentration (MIC) Interpretive Standards" in the CLSI document, except for amikacin and ciprofloxacin. For amikacin, we used $10 \mu \mathrm{g}$ disks and 11 of $13 \mathrm{~S}$. Dublin isolates were susceptible to the $10 \mu \mathrm{g}$ disk, while 2 were intermediate-resistant. This is in agreement with the indicated minimal inhibitory concentration (MIC), which is $\leq 16 \mu \mathrm{g} / \mathrm{mL}$. However, for ciprofloxacin, the disk content in the document is $5 \mu \mathrm{g}$; we used $30 \mu \mathrm{g}$ disks, but only $3 \mathrm{~S}$. Dublin isolates were susceptible, while 10 were intermediate-resistant (Table 3 ). Indeed, the MIC of ciprofloxacin indicated in the document is $\leq 1 \mu \mathrm{g} / \mathrm{mL}$. Of all the isolates, $11(85 \%)$ were considered to be multiple drugresistant to two or more antibiotics (Table 3 ). No isolate was susceptible to all tested antibiotics.

\section{Discussion}

Studies on the prevalence and antibiotic susceptibility of Salmonella isolates from Addis Ababa abattoir are scarce. The presence of several Salmonella serotypes that can cause disease in both animals and humans, the high chance for zoonotic transmissions, and the fact that several antibiotic classes are used in both veterinary and human medicine dictate the need for continued surveillance of Salmonella in foods and the environment. This study found low-level contamination of sampled tissues and swabs with $S$. Dublin that, however, can be significant sources for further contamination during processing and handling.

Carcass contamination with Salmonella is of special public health significance for a country like Ethiopia, where consumption of raw and undercooked meat is common in most areas of Ethiopia. Contamination is likely to be further amplified as meat passes through various chains until it reaches the final consumers. A recent meta-analysis of Salmonella contamination in raw animal products in Ethiopia reported that $S$. Dublin was the most frequently isolated serotype in beef and revealed that the odds of contamination were $>2$ fold higher in retail markets than in abattoirs [44].

Although $S$. Dublin is known to be cattle-adapted, it can infect other domestic animals including ovines [14, 15, 45]. It primarily affects the mammary glands of cows and can be shed into milk subsequently causing infection in humans 
TABLE 3: Antibiotic disk diffusion susceptibility test results for bovine and ovine isolates of Salmonella.

\begin{tabular}{|c|c|c|c|c|c|c|c|c|c|c|c|c|c|}
\hline Sample & Amik & Amox/clav & Amp & Ceftr & Chl & Cipro & Gent & Kan & $\mathrm{Nal}$ & Nitrof & Strep & Tri/sul & Tet \\
\hline B.17 & $S$ & $\mathrm{R}$ & $S$ & I & $S$ & $S$ & $S$ & $S$ & $S$ & $S$ & $\mathrm{R}$ & $S$ & $S$ \\
\hline B. 21 & $S$ & $\mathrm{R}$ & $\mathrm{R}$ & $\mathrm{R}$ & $\mathrm{R}$ & S & I & $\mathrm{R}$ & $\mathrm{R}$ & $\mathrm{R}$ & $\mathrm{R}$ & S & $\mathrm{R}$ \\
\hline B. 48 & S & $\mathrm{R}$ & S & S & S & I & S & S & S & S & $\mathrm{R}$ & S & S \\
\hline B.61 & S & $\mathrm{R}$ & $\mathrm{R}$ & $\mathrm{R}$ & $\mathrm{R}$ & I & S & $\mathrm{R}$ & I & S & $\mathrm{R}$ & S & $\mathrm{R}$ \\
\hline B.S.1 & S & I & S & I & $S$ & I & S & S & $S$ & $S$ & $\mathrm{R}$ & $\mathrm{R}$ & S \\
\hline B.S.12 & S & $\mathrm{R}$ & S & S & S & I & S & S & S & $\mathrm{R}$ & $\mathrm{R}$ & S & $\mathrm{S}$ \\
\hline B.S.13 & S & $\mathrm{R}$ & $S$ & I & S & I & S & $S$ & S & I & $\mathrm{R}$ & I & $S$ \\
\hline B.S.23 & I & I & S & S & S & I & S & S & S & S & $\mathrm{R}$ & S & $S$ \\
\hline M.6 & S & $\mathrm{R}$ & S & S & S & S & S & S & S & I & $\mathrm{R}$ & S & $S$ \\
\hline M.26 & $S$ & I & S & S & $S$ & I & S & S & $S$ & I & $\mathrm{R}$ & S & S \\
\hline M.28 & $S$ & $\mathrm{R}$ & S & S & $S$ & I & S & I & S & $\mathrm{R}$ & $\mathrm{R}$ & $\mathrm{R}$ & $S$ \\
\hline M.72 & $S$ & $\mathrm{R}$ & $S$ & $S$ & $S$ & I & $S$ & I & $S$ & $\mathrm{R}$ & $\mathrm{R}$ & I & $S$ \\
\hline S.S.16 & I & $\mathrm{R}$ & S & I & $S$ & I & $S$ & $S$ & I & $\mathrm{R}$ & $\mathrm{R}$ & $\mathrm{R}$ & $\mathrm{S}$ \\
\hline
\end{tabular}

Amik: amikacin; Amox/clav: amoxicillin/clavulanic acid; Amp: ampicillin; Ceftr: ceftriaxone; Chl: chloramphenicol; Cipro: ciprofloxacin; Gent: gentamycin; Kan: kanamycin, Nal: nalidixic acid; Nitro: nitrofurantoin; Strep: streptomycin; Tri/sul: trimethoprim/sulphamethoxazole; Tet: tetracycline; S: susceptible; R: resistant; I: intermediate-resistant.

consuming unpasteurized dairy products [46-49]. Eguale et al. [50] reported the finding of $S$. Dublin in dairy cattle in Ethiopia. In Ethiopia, consumption of raw milk is very common largely because of the impracticability in most cases of boiling milk before consumption. Many people in Ethiopia also recognize the richness of liver in nutrients and consume it raw with hot pepper or spices. Thus, it is reasonable to assume that meat, milk, and liver can serve as vehicles for extensive transmission of Salmonella to humans.

S. Dublin can cause disease in animals and invasive disease and mortality in humans [51-53]. Nontyphoidal Salmonella, including S. Dublin, cause bacteremia, bloodstream, and other infections in humans in sub-Saharan Africa $[10,11]$. Virulence plasmids are found in S. Dublin and other Salmonella serovars [54-56]. S. Dublin causes enteritis by inducing infiltration of neutrophils into the intestinal epithelium, induction of inflammatory responses, and fluid secretion mediated by secretion systems that translocate secreted effector proteins into eukaryotic cells [52, 57]. S. Dublin is considered highly pathogenic to humans, especially in immunocompromised individuals [12-14].

Resistance of bovine and ovine isolates of Salmonella to two or more major antibiotics was observed in 11 (84.6\%) of isolates in this study. This resistance to antibiotics has significant importance because these antibiotics are also commonly used in human medicine in Ethiopia. For example, ciprofloxacin, nalidixic acid, and ceftriaxone are indicated against bacillary dysentery; chloramphenicol, trimethoprim/ sulfamethoxazole, and ciprofloxacin are indicated against gastroenteritis; the latter two drugs are also used to treat cholera; amoxicillin, gentamycin, and ceftriaxone are among the agents used to treat pneumonia; trimethoprim/sulfamethoxazole and amoxicillin/clavulanic acid are used against sinusitis [58]. Moreover, prescriptions are usually made without prior isolation and drug susceptibility testing of infectious agents. Furthermore, the potential for horizontal transmission of resistance traits and other virulence factors or plasmids from Salmonella to other microbes, including within the human gut, may exist. Resistance traits in Salmonella can be genetically determined and may involve chromosomal mutations or may be plasmid-mediated and may be exchanged with other Enterobacteriaceae [59-62]. The magnitude of all these is not known in the prevailing conditions in Ethiopia since misdiagnosis and underdiagnosis can be common and prescriptions are virtually empirical. Large-scale intensive farming combined with use of antibiotics (which is empirical in most developing countries) in animals is expected to increase in the coming decades, thereby promoting on-farm selection of antibiotic-resistant strains and markedly increasing the human health risks associated with consumption of contaminated meat products $[24,25]$.

To our knowledge, this is the first study to have complete (100\%) resistance to streptomycin in all studied isolates being reported among bovine or ovine isolates of Salmonella in Ethiopia. Thus, it is of significant concern, since our study involves all randomly selected samples. Resistance of Salmonella from food items, animals, and humans to streptomycin was reported by several studies in Ethiopia [35, 36, $50,63-65]$, but the level of resistance to streptomycin ranged from 46 to $86 \%$. This extremely high level of resistance of Salmonella and other pathogens (e.g., similar high level resistance to streptomycin has also been reported in Ethiopian tuberculosis patients, including in newly diagnosed patients ([66] and references therein)) to streptomycin in Ethiopia should be cause for high concern as it might also cause crossresistance to other drugs with similar mechanism of action. Gentamycin was effective on almost all isolates in this study, similar to that of Garedew et al. [28] and Alemu and Zewde [67] who analyzed Salmonella from slaughtered bovines, raw meat, and swab samples from butcher shops' utensils and meat handlers.

This study has some limitations. Inclusion of possible Salmonella isolates from humans in the abattoir and the internal abattoir environment would have strengthened this 
study. The results of this study are indicative of Salmonella risk in meat from the abattoir, but more detailed studies should be conducted including possible routes of transmission of Salmonella as well as amplification of antibiotic resistance transfer.

\section{Conclusions}

The present study indicated detection of Salmonella from healthy slaughtered bovine and ovine samples at Addis Ababa abattoir enterprise with an overall prevalence of $4.64 \%$. The results of the present study indicate poor evisceration process and hygienic practices of workers, which could result in the contamination of carcass and cross contamination from positive animals. This study also revealed high resistance of Salmonella to commonly used antibiotics. Contamination with Salmonella can be further amplified when one considers the possibilities for more contamination as meat passes through the many steps from slaughterhouse to the final consumer [29, 68]. Hygiene status must be enhanced to minimize cross contamination of Salmonella from utensils, cutting boards, and knifes as well as from abattoir workers who are involved in the slaughtering process. Even if $S$. Dublin were a bovine pathogen only, it would still be a highly significant problem to the economy and livelihood of people in general and farmers in particular. Proper decontamination and disinfection measures should be enhanced including at the entrance to slaughterhouses to reduce contamination. Further recommended measures to control contamination include carcass trimming to remove visible contamination, washes using ambient or hot water, organic acids, and other chemicals, as well as hide dehairing and frequent hand washes and disinfection. To minimize the risk of cross contamination and food-borne infections caused by Salmonella spp., control measures along the meat processing chain, namely, slaughterhouses, meat processing plants, distributors, and consumers, should be undertaken [69]. These measures should be introduced or practiced regularly at the Addis Ababa abattoir. Care should be taken in selecting antibiotics to treat Salmonella infection in animals and humans to avoid subtherapeutic levels of (appropriate) antibiotics and use of inappropriate antibiotics (e.g., antibiotic(s) to which an infectious agent may be resistant). Finally, larger studies are recommended to elucidate the magnitude of infection of animals with Salmonella, the extent of zoonotic transmissions, and the antibiotic resistance problem. A Pubmed search using combinations of key words such as "Addis Ababa", "Abattoir", "Salmonella", "Bovine", "Antibiotic Resistance" returned no published articles. We hope the results of this study could serve as basis for initiation of further and larger studies in Ethiopia that will shed light on the types, levels, frequency, and appropriateness of antibiotic use in farm animals as well as transmission of pathogens and antibiotic resistance from animals to humans, which will be important for the benefit of animal and human health. Such studies require implementation of carefully designed experiments to determine and trace associations between causes and effects and effects of subtherapeutic levels of antibiotics [70]. Thus, collaborative efforts with combined inputs of both expertise and resources would be needed.

\section{Competing Interests}

The authors declare that there are no competing interests regarding the publication of this paper.

\section{Authors' Contributions}

Abe Kebede, Jelalu Kemal, and Solomon Habte Mariam designed the work; Abe Kebede and Haile Alemayehu prepared the samples and performed all the experiments and laboratory works. Jelalu Kemal and Solomon Habte Mariam analyzed all the data. The paper was written by Jelalu Kemal and Solomon Habte Mariam. All authors revised and approved the final paper.

\section{Acknowledgments}

The authors would like to acknowledge Haramaya University, College of Veterinary Medicine, for its facilitation and Aklilu Lemma Institute of Pathobiology for unreserved material and reagent supply for the study. They thank Tadesse Eguale, Gitanjali Arya, and the OIE Salmonella Reference Laboratory of the Public Health Agency of Canada for serotyping the Salmonella isolates.

\section{References}

[1] World Health Organization, "Salmonella (non-typhoidal)," Fact Sheet 139, WHO, 2013, http://www.who.int/mediacentre/ factsheets/fs139/en/.

[2] S. Hoffmann, M. B. Batz, and J. G. Morris Jr., "Annual cost of illness and quality-adjusted life year losses in the united states due to 14 foodborne pathogens," Journal of Food Protection, vol. 75, no. 7, pp. 1292-1302, 2012.

[3] C. F. Pui, W. C. Wong, L. C. Chai et al., "Salmonella: a foodborne pathogen," International Food Research Journal, vol. 18, no. 2, pp. 465-473, 2011.

[4] M. D. Kirk, S. M. Pires, and R. E. Black, "World Health Organization estimates of the global and regional disease burden of 22 foodborne bacterial, protozoal, and viral diseases 2010: a data synthesis," PLoS Medicine, vol. 12, no. 12, Article ID e1001921, 2015.

[5] N. A. Feasey, G. Dougan, R. A. Kingsley, R. S. Heyderman, and M. A. Gordon, "Invasive non-typhoidal Salmonella disease: an emerging and neglected tropical disease in Africa," The Lancet, vol. 379, no. 9835, pp. 2489-2499, 2012.

[6] C. R. Braden, "Salmonella enterica serotype enteritidis and eggs: a national epidemic in the United States," Clinical Infectious Diseases, vol. 43, no. 4, pp. 512-517, 2006.

[7] B. R. Jackson, P. M. Griffin, D. Cole, K. A. Walsh, and S. J. Chai, "Outbreak-associated Salmonella enterica serotypes and food commodities, United States, 1998-2008," Emerging Infectious Diseases, vol. 19, no. 8, pp. 1239-1244, 2013.

[8] C. Bayer, H. Bernard, R. Prager et al., "An outbreak of Salmonella Newport associated with mung bean sprouts in Germany and the Netherlands, October to November 2011," Eurosurveillance, vol. 19, no. 1, 2014. 
[9] Centers for Disease Control and Prevention, "The Food Production Chain-How Food Gets Contaminated," Foodborne Outbreaks, Investigating Outbreaks, 2015, http://www.cdc.gov.

[10] S. M. Tennant, S. Diallo, H. Levy et al., "Identification by PCR of non-typhoidal Salmonella enterica serovars associated with invasive infections among febrile patients in Mali," PLoS Neglected Tropical Diseases, vol. 4, no. 3, article e621, 2010.

[11] H. M. Al-Emran, R. Krumkamp, D. M. Dekker et al., "Validation and identification of invasive Salmonella serotypes in subsaharan africa by multiplex polymerase chain reaction," Clinical Infectious Diseases, vol. 62, supplement 1, pp. S80-S82, 2016.

[12] W. C. Levine, J. W. Buehler, N. H. Bean, and R. V. Tauxe, "Epidemiology of nontyphoidal Salmonella bacteremia during the human immunodeficiency virus epidemic," Journal of Infectious Diseases, vol. 164, no. 1, pp. 81-87, 1991.

[13] C. A. MacLennan, J. J. Gilchrist, M. A. Gordon et al., "Dysregulated humoral immunity to nontyphoidal Salmonella in HIVinfected African adults," Science, vol. 328, no. 5977, pp. 508-512, 2010.

[14] R. K. Selander, N. H. Smith, J. Li et al., "Molecular evolutionary genetics of the cattle-adapted serovar Salmonella dublin," Journal of Bacteriology, vol. 174, no. 11, pp. 3587-3592, 1992.

[15] S. Uzzau, D. J. Brown, T. Wallis et al., "Host adapted serotypes of Salmonella enterica," Epidemiology and Infection, vol. 125, no. 2, pp. 229-255, 2000.

[16] M. P. Stevens, T. J. Humphrey, and D. J. Maskell, "Molecular insights into farm animal and zoonotic Salmonella infections," Philosophical Transactions of the Royal Society of London B: Biological Sciences, vol. 364, no. 1530, pp. 2709-2723, 2009.

[17] K. Hoelzer, A. I. M. Switt, and M. Wiedmann, "Animal contact as a source of human non-typhoidal salmonellosis," Veterinary Research, vol. 42, no. 1, article 34, 2011.

[18] B. G. Meerburg and A. Kijlstra, "Role of rodents in transmission of Salmonella and Campylobacter," Journal of the Science of Food and Agriculture, vol. 87, no. 15, pp. 2774-2781, 2007.

[19] M. N. Skov, J. J. Madsen, C. Rahbek et al., "Transmission of Salmonella between wildlife and meat-production animals in Denmark," Journal of Applied Microbiology, vol. 105, no. 5, pp. 1558-1568, 2008.

[20] D. G. White, S. Zhao, R. Sudler et al., "The isolation of antibioticresistant Salmonella from retail ground meats," The New England Journal of Medicine, vol. 345, no. 16, pp. 1147-1154, 2001.

[21] E. J. Threlfall, "Antimicrobial drug resistance in Salmonella: problems and perspectives in food- and water-borne infections," FEMS Microbiology Reviews, vol. 26, no. 2, pp. 141-148, 2002.

[22] J. Kemal, "A review on the public health importance of bovine salmonellosis," Journal of Veterinary Science and Technology, vol. 5, no. 2, Article ID 1000175, 2014.

[23] R. Cabrera, J. Ruiz, F. Marco et al., "Mechanism of resistance to several antimicrobial agents in Salmonella clinical isolates causing traveler's diarrhea," Antimicrobial Agents and Chemotherapy, vol. 48, no. 10, pp. 3934-3939, 2004.

[24] H. C. Wegner, F. M. Aarestrup, P. Gerner-Smidt, and F. Bager, "Transfer of antibiotic resistant bacteria from animals to man," Acta Veterinarica Scandinavsca, supplement 92, pp. 51-57, 1999.

[25] T. P. Van Boeckel, C. Brower, M. Gilbert et al., "Global trends in antimicrobial use in food animals," Proceedings of the National Academy of Sciences of the United States of America, vol. 112, no. 18, pp. 5649-5654, 2015.
[26] S. Omulo, S. M. Thumbi, M. K. Njenga, and D. R. Call, "A review of 40 years of enteric antimicrobial resistance research in Eastern Africa: what can be done better?" Antimicrobial Resistance and Infection Control, vol. 4, article 1, 2015.

[27] W. S. Darwish, E. A. Eldaly, M. T. El-Abbasy, Y. Ikenaka, S. Nakayama, and M. Ishizuka, "Antibiotic residues in food: the African scenario," Japanese Journal of Veterinary Research, vol. 61, pp. S13-S22, 2013.

[28] L. Garedew, Z. Hagos, Z. Addis, R. Tesfaye, and B. Zegeye, "Prevalence and antimicrobial susceptibility patterns of Salmonella isolates in association with hygienic status from butcher shops in Gondar town, Ethiopia," Antimicrobial Resistance and Infection Control, vol. 4, no. 1, article 21, 2015.

[29] C. Nyeleti, G. Hildebrandt, J. Kleer, and B. Molla, "Prevalence of Salmonella in Ethiopian cattle and minced beef," Berliner und Munchener Tierarztliche Wochenschrift, vol. 113, no. 11-12, pp. 431-434, 2000.

[30] D. Alemayehu, B. Molla, and A. Muckle, "Prevalence and antimicrobial resistance pattern of Salmonella isolates from apparently healthy slaughtered cattle in Ethiopia," Tropical Animal Health and Production, vol. 35, no. 4, pp. 309-319, 2003.

[31] A. Kindu and M. Addis, "A survey on Salmonella infection among chicken flocks in Jimma town, Ethiopia," African Journal of Microbiology Research, vol. 7, no. 14, pp. 1239-1245, 2013.

[32] G. Tadesse and T. S. Tessema, "A meta-analysis of the prevalence of Salmonella in food animals in Ethiopia," BMC Microbiology, vol. 14, article 270, 2014.

[33] O. Olsvik, H. Sørum, K. Birkness et al., "Plasmid characterization of Salmonella typhimurium transmitted from animals to humans," Journal of Clinical Microbiology, vol. 22, no. 3, pp. 336338, 1985.

[34] S. W. M. Hendriksen, K. Orsel, J. A. Wagenaar, A. Miko, and E. Van Duijkeren, "Animal-to-human transmission of Salmonella Typhimurium DT104A variant," Emerging Infectious Diseases, vol. 10, no. 12, pp. 2225-2227, 2004.

[35] W. Molla, B. Molla, D. Alemayehu, A. Muckle, L. Cole, and E. Wilkie, "Occurrence and antimicrobial resistance of Salmonella serovars in apparently healthy slaughtered sheep and goats of central Ethiopia," Tropical Animal Health and Production, vol. 38, no. 6, pp. 455-462, 2006.

[36] E. Zewdu and P. Cornelius, "Antimicrobial resistance pattern of Salmonella serotypes isolated from food items and personnel in Addis Ababa, Ethiopia," Tropical Animal Health and Production, vol. 41, no. 2, pp. 241-249, 2009.

[37] M. Thrusfield, Veterinary Epidemiology, Blackwell Science, London, UK, 3rd edition, 2007.

[38] International Standards Organization, "Microbiology of food and animal feeding stuffs: carcass sampling for microbiological analysis," Tech. Rep. ISO-17604, International Standards Organization, Geneva, Switzerland, 2003.

[39] International Organization for Standardization (ISO), "Microbiology of food and animal feeding stuffs: horizontal method for the detection of Salmonella spp.," ISO, Geneva, Switzerland, pp. 511-525, 2002.

[40] P. R. Murray and Y. Shea, Guide to Clinical Microbiology, American Society for Microbiology, Washington, DC, USA, 3rd edition, 2004.

[41] R. R. MacFadden, Biochemical Tests for Identification of Medical Bacteria, Lippincott Williams \& Wilkins, Philadelphia, Pa, USA, 2000 . 
[42] N. D. Cohen, H. L. Neibergs, E. D. McGruder et al., "Genusspecific detection of salmonellae using the polymerase chain reaction (PCR)," Journal of Veterinary Diagnostic Investigation, vol. 5, no. 3, pp. 368-371, 1993.

[43] Clinical Laboratory Standards Institute (CLSI), "Performance standards for antimicrobial susceptibility testing twenty second informational supplement," Document M100-S22, Clinical Laboratory Standards Institute, Wayne, Pa, USA, 2012.

[44] G. Tadesse and E. Z. Gebremedhin, "Prevalence of Salmonella in raw animal products in Ethiopia: a meta-analysis," $B M C$ Research Notes, vol. 8, article 163, 2015.

[45] M. S. Bulgin, "Salmonella dublin: what veterinarians should know," Journal of the American Veterinary Medical Association, vol. 182, no. 2, pp. 116-118, 1983.

[46] S. Benson Werner, G. L. Humphrey, and I. Kamei, "Association between raw milk and human Salmonella Dublin infection," British Medical Journal, vol. 2, no. 6184, pp. 238-241, 1979.

[47] B. P. Smith, D. G. Oliver, P. Singh et al., "Detection of Salmonella dublin mammary gland infection in carrier cows, using an enzyme-linked immunosorbent assay for antibody in milk or serum," American Journal of Veterinary Research, vol. 50, no. 8, pp. 1352-1360, 1989.

[48] S. J. Spier, B. P. Smith, J. W. Tyler, J. S. Cullor, G. W. Dilling, and L. Da Pfaff, "Use of ELISA for detection of immunoglobulins G and M that recognize Salmonella dublin lipopolysaccharide for prediction of carrier status in cattle," American Journal of Veterinary Research, vol. 51, no. 12, pp. 1900-1904, 1990.

[49] J. S. Cullor, "Risks and prevention of contamination of dairy products," Revue Scientifique et Technique, vol.16, no. 2, pp. 472481, 1997.

[50] T. Eguale, E. Engidawork, W. A. Gebreyes et al., "Fecal prevalence, serotype distribution and antimicrobial resistance of Salmonellae in dairy cattle in central Ethiopia," BMC Microbiology, vol. 16, no. 1, article 20, 2016.

[51] D. M. Heithoff, W. R. Shimp, P. W. Lau et al., "Human Salmonella clinical isolates distinct from those of animal origin," Applied and Environmental Microbiology, vol. 74, no. 6, pp. 1757-1766, 2008.

[52] T. F. Jones, L. A. Ingram, P. R. Cieslak et al., "Salmonellosis outcomes differ substantially by serotype," Journal of Infectious Diseases, vol. 198, no. 1, pp. 109-114, 2008.

[53] L. Yim, S. Sasías, A. Martínez et al., "Repression of flagella is a common trait in field isolates of Salmonella enterica serovar Dublin and is associated with invasive human infections," Infection and Immunity, vol. 82, no. 4, pp. 1465-1476, 2014.

[54] T. S. Wallis, S. M. Paulin, J. S. Plested, P. R. Watson, and P. W. Jones, "The Salmonella dublin virulence plasmid mediates systemic but not enteric phases of Salmonellosis in cattle," Infection and Immunity, vol. 63, no. 7, pp. 2755-2761, 1995.

[55] F. A. Norris, M. P. Wilson, T. S. Wallis, E. E. Galyov, and P. W. Majerus, "SopB, a protein required for virulence of Salmonella dublin, is an inositol phosphate phosphatase," Proceedings of the National Academy of Sciences of the United States of America, vol. 95, no. 24, pp. 14057-14059, 1998.

[56] J. Fierer and D. G. Guiney, "Diverse virulence traits underlying different clinical outcomes of Salmonella infection," The Journal of Clinical Investigation, vol. 107, no. 7, pp. 775-780, 2001.

[57] E. E. Galyov, M. W. Wood, R. Rosqvist et al., "A secreted effector protein of Salmonella dublin is translocated into eukaryotic cells and mediates inflammation and fluid secretion in infected ileal mucosa," Molecular Microbiology, vol. 25, no. 5, pp. 903-912, 1997.
[58] Drug Administration and Control Authority of Ethiopia, Standard Treatment Guidelines for District Hospitals, Drug Administration and Control Authority of Ethiopia, Addis Ababa, Ethiopia, 2004.

[59] V. Miriagou, P. T. Tassios, N. J. Legakis, and L. S. Tzouvelekis, "Expanded-spectrum cephalosporin resistance in non-typhoid Salmonella," International Journal of Antimicrobial Agents, vol. 23, no. 6, pp. 547-555, 2004.

[60] P. Antunes, J. Machado, J. C. Sousa, and L. Peixe, "Dissemination amongst humans and food products of animal origin of a Salmonella typhimurium clone expressing an integron-borne OXA-30 $\beta$-lactamase," Journal of Antimicrobial Chemotherapy, vol. 54, no. 2, pp. 429-434, 2004.

[61] S. Domingues, K. Harms, W. Florian Fricke, P. J. Johnsen, G. J. da Silva, and K. M. Nielsen, "Natural transformation facilitates transfer of transposons, integrons and gene cassettes between bacterial species," PLoS Pathogens, vol. 8, no. 8, Article ID e1002837, 2012.

[62] J. A. Crump, M. Sjölund-Karlsson, M. A. Gordon, and C. M. Parry, "Epidemiology, clinical presentation, laboratory diagnosis, antimicrobial resistance, and antimicrobial management of invasive Salmonella infections," Clinical Microbiology Reviews, vol. 28, no. 4, pp. 901-937, 2015.

[63] B. Molla, J. Kleer, and H.-J. Sinell, "Antibiotic resistance pattern of foodborne Salmonella isolates in Addis Ababa (Ethiopia)," Berliner und Munchener Tierarztliche Wochenschrift, vol. 112, no. 2, pp. 41-43, 1999.

[64] G. Tadesse, "A meta-analysis of the proportion of animal Salmonella isolates resistant to drugs used against human salmonellosis in Ethiopia," BMC Infectious Diseases, vol. 15, article 84, 2015.

[65] T. Eguale, W. A. Gebreyes, D. Asrat, H. Alemayehu, J. S. Gunn, and E. Engidawork, "Non-typhoidal Salmonella serotypes, antimicrobial resistance and co-infection with parasites among patients with diarrhea and other gastrointestinal complaints in Addis Ababa, Ethiopia," BMC Infectious Diseases, vol. 15, article 497, 2015.

[66] M. Maru, S. H. Mariam, T. Airgecho, E. Gadissa, and A. Aseffa, "Prevalence of tuberculosis, drug susceptibility testing, and genotyping of mycobacterial isolates from pulmonary tuberculosis patients in Dessie, Ethiopia," Tuberculosis Research and Treatment, vol. 2015, Article ID 215015, 10 pages, 2015.

[67] S. Alemu and B. M. Zewde, "Prevalence and antimicrobial resistance profiles of Salmonella enterica serovars isolated from slaughtered cattle in Bahir Dar, Ethiopia," Tropical Animal Health and Production, vol. 44, no. 3, pp. 595-600, 2012.

[68] B. K. Khen, O. A. Lynch, J. Carroll, D. A. Mcdowell, and G. Duffy, "prevalence and characteristics of Salmonella in the beef chain in the Republic of Ireland," Zoonoses and Public Health, vol. 61, no. 8, pp. 534-536, 2014.

[69] FAO, "Interventions for the control of nontyphoidal Salmonella spp. in beef and pork," Report of a Joint FAO/WHO Expert Meeting, FAO, Rome, Italy, 2015.

[70] Committee to Study the Human Health Effects of Subtherapeutic Antibiotic Use in Animal Feeds, Division of Medical Sciences, National Research Council, The Effects on Human Health of Subtherapeutic Use of Antimicrobials in Animal Feeds, 1980, http://www.nap.edu/catalog/21.html. 

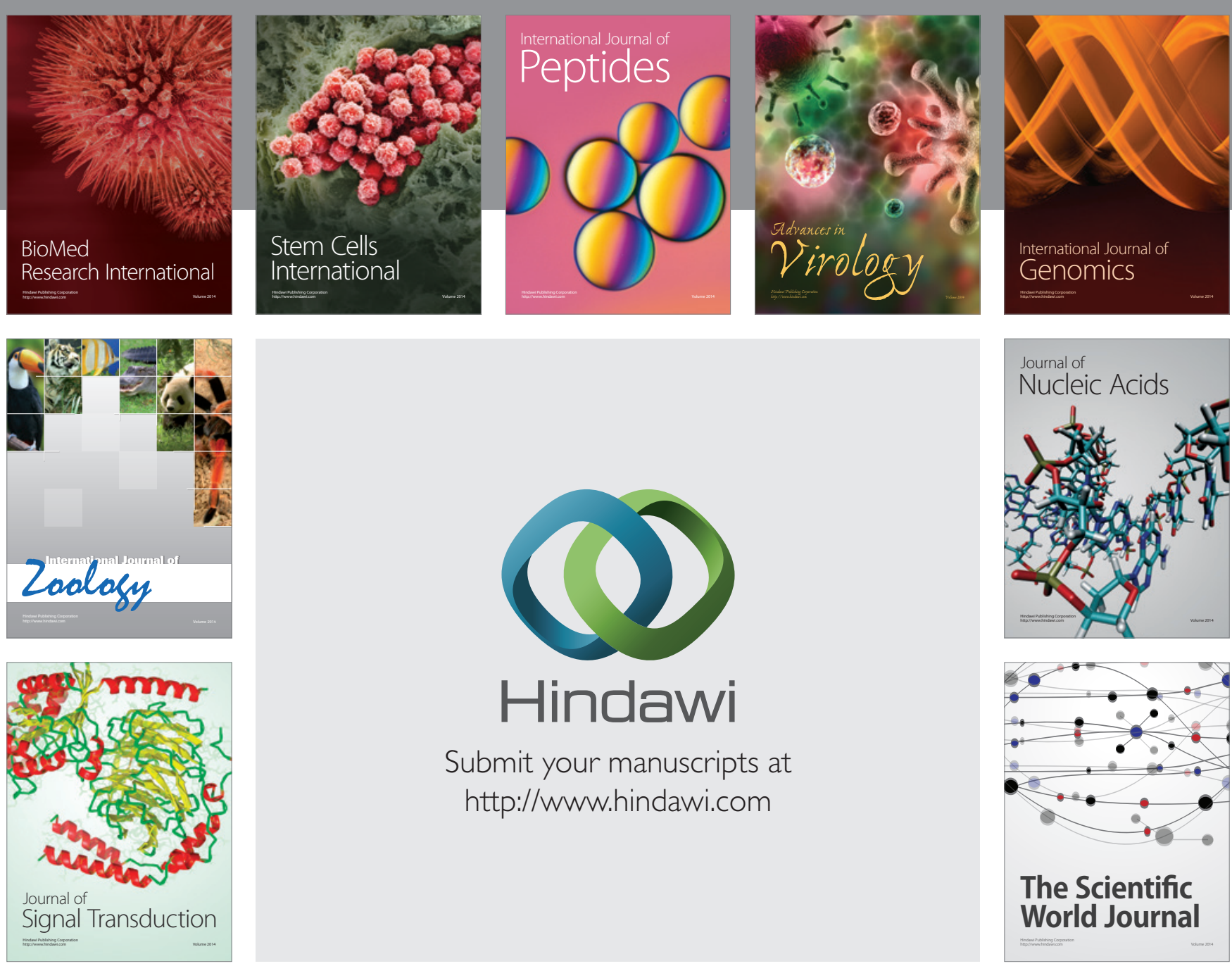

Submit your manuscripts at

http://www.hindawi.com
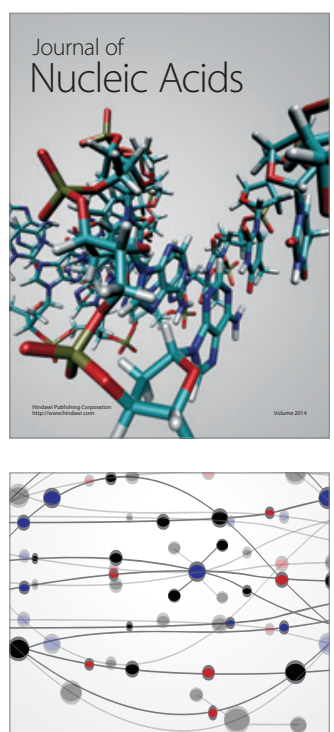

The Scientific World Journal
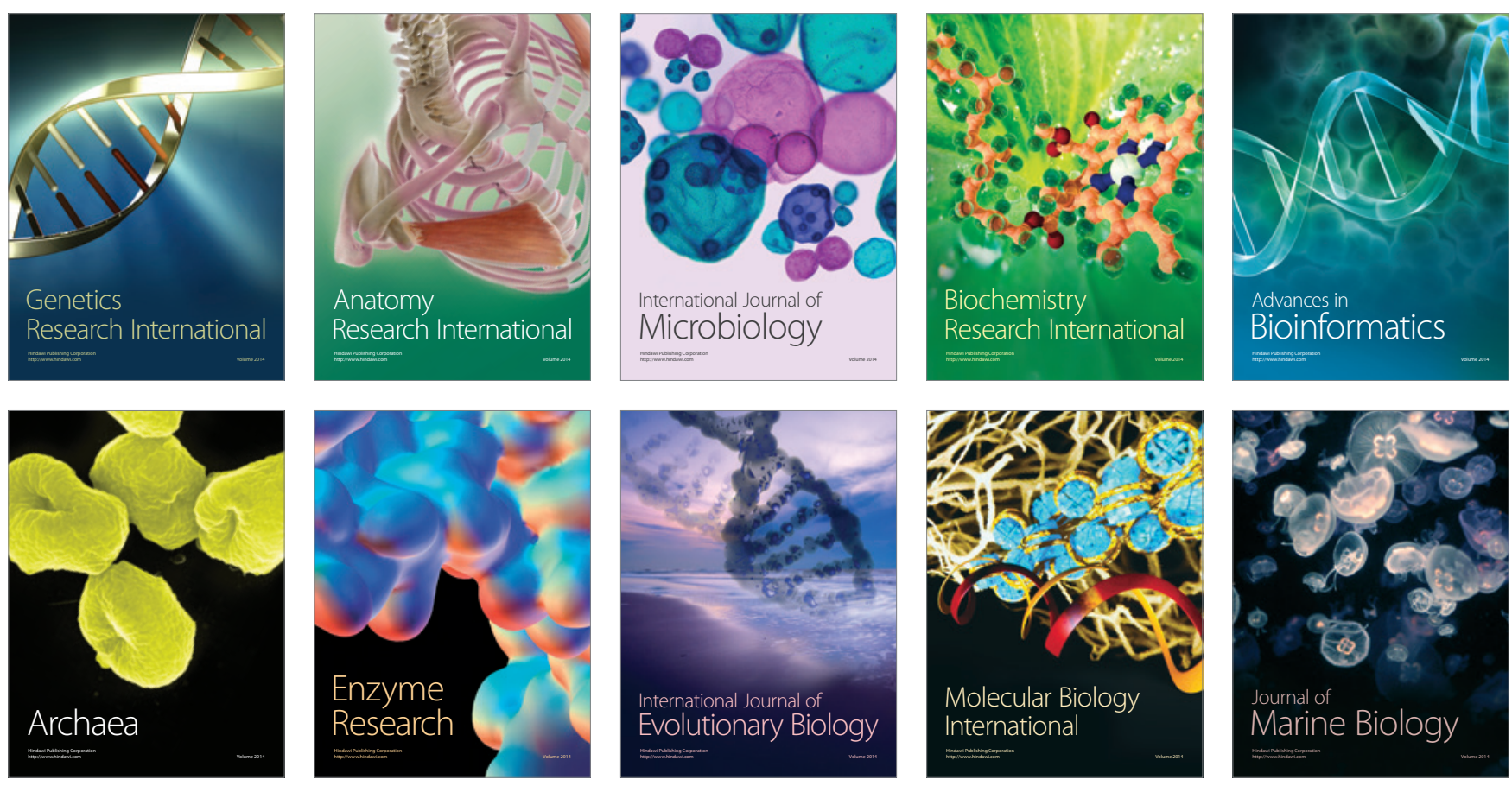